\title{
On Network Interference Management
}

\author{
Aleksandar Jovičić, Hua Wang and Pramod Viswanath *†
}

March 3, 2008

\begin{abstract}
We study two building-block models of interference-limited wireless networks, motivated by the problem of joint Peer-to-Peer and Wide Area Network design. In the first case, a single "long-range" transmitter interferes with multiple parallel "short-range" transmissions, and, in the second case, multiple short-range transmitters interfere with a single long-range receiver. We identify the maximal degree-of-freedom region of the former network and show that multilevel superposition coding by the long-range transmitter performs optimally. Moreover, a simple power control strategy, performed by the long-range transmitter, achieves a region that is within one bit of the capacity region, under certain channel conditions. For the latter network, we show that short-range transmitter power control is degreeof-freedom optimal under certain channel conditions.
\end{abstract}

\section{Introduction}

The convergence of heterogenous radio devices and services in the unlicensed as well as the legacy-operator bands has created a need for highly spectrally efficient communication in the presence of multiuser interference bearing spatially non-uniform statistics. The increased popularity of short-range peer-to-peer communication (Bluetooth and WiFi, for instance), along with the more traditional demand for mobile long-range WAN access, is leading up to a clash of scales and a possibility of throughput degradation in both types of networks if they are to occupy the same spectrum.

Practical peer-to-peer protocols as well as cellular wide area network interference management technologies have traditionally centered around two fundamental basic schemes: orthogonalization and full-reuse. Orthogonalization divides the total degrees of freedom (time or frequency) to the different users, while, at the other extreme, full reuse allows each point-to-point communication to take place over the same time and frequency band and multiuser interference is treated as noise.

${ }^{*}$ A. Jovičić is with Qualcomm Flarion Technologies, Bridgewater, NJ. H. Wang and P. Viswanath are with the department of Electrical and Computer Engineering at the University of Illinois at UrbanaChampaign. Email: ajovicic@qualcomm.com, hwang@uiuc.edu and pramodv@uiuc.edu

${ }^{\dagger}$ This research was supported in part by the National Science Foundation under grant CCR-0312413 and a grant from Motorola Inc. as part of the Motorola Center for Communication. 
In this paper, we study the problem of joint short- and long-range network design from an information-theoretic standpoint. More specifically, our focus is on finding optimal interference management schemes for two specific wireless network topologies: the manyreceiver, single-interferer and the many-interferer, single receiver networks. These two examples are illustrated in Fig. 1.

In the first example, only one transmitter is creating interference to the other receivers as it communicates with its intended receiver. This situation could correspond to a setting in which one long-range WAN transmission such as a cellular uplink is taking place over the same time and frequency bands as multiple local short-range peer-to-peer transmissions. The long-range transmitter is typically more powerful than the short-range radios and hence will generate significant interference. At the same time, the shortrange transmissions are not powerful enough to cause interference among themselves. In the second example, the short-range peer-to-peer communications create interference for the long-range receiver. This could take place when a WAN downlink is experiencing interference from a group of neighboring peer-to-peer transmissions.

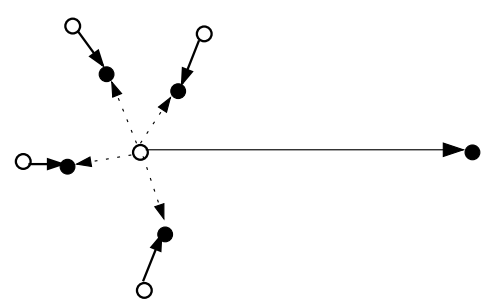

(a)

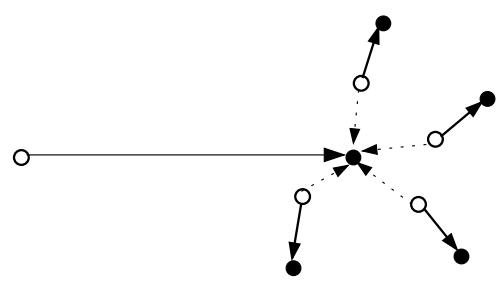

(b)

Figure 1: The many-receiver, single-interferer network in (a) and the single-receiver, many-interferer network in (b). The transmitters are denoted by empty circles and receivers by filled circles. The solid arrows are intended communication links and the dotted arrows represent the interference.

Our main results are approximations to the capacity regions of the two networks illustrated in Fig. 1. For the single-interferer, many-receiver network of Fig. 1 (a), we show that a simple power-control strategy achieves a region that is within one bit of the capacity region of the network, in a "weak interference" regime. Our result is an extension and a strengthening of the two-user "Z-channel" result of [4].

Furthermore, we study the behavior of the network in the asymptotic interference limited regime (introduced in [4]) in which the transmit and received signal-to-noise 
ratios (SNRs) approach infinity while keeping fixed the ratios of the received SNRs (in decibels) of the signals of interest to the transmitted SNR (in decibles), as well as the ratios of the received SNRs of the interference to the transmitted SNR. In this regime, we obtain a complete characterization of the degree-of-freedom region of the network of Fig. 1 (a). We show that the communication scheme that achieves all points in this region is multilevel superposition coding at the long-range transmitter and successive interference decoding and cancellation at the short-range receivers. For the network of Fig. 1 (b), we identify the degree-of-freedom region in this same regime, and we show that the optimal scheme is transmit power control by the short-range transmitters.

This paper is organized as follows: in Section 2 we introduce the channel model and the definitions of the approximations to the capacity region. In Section 3 we state our main results which are proved in Section 4. Finally, we discuss the extension of the classical result on the capacity in the "strong-interference" regime in Section 5.

\section{Preliminaries}

\subsection{The channel model}

Suppose that there are $k$ short-range users in both networks of Fig. 1. We will refer to the long-range user as "user 0" and short-range user $i$ simply as "user $i$ ", for $i=1,2, \ldots, k$. The network channels can be represented by the following equations, which are also depicted in Fig. 2:

Network (a) of Fig. 1:

$$
\begin{aligned}
& Y_{0}=h_{00} X_{0}+Z_{0}, \\
& Y_{i}=h_{0 i} X_{0}+h_{i i} X_{i}+Z_{i}
\end{aligned}
$$

Network (b) of Fig. 1:

$$
\begin{array}{r}
Y_{0}=h_{00} X_{0}+\sum_{i=1}^{k} h_{i 0} X_{i}+Z_{0}, \\
Y_{i}=h_{i i} X_{i}+Z_{i},
\end{array}
$$

where $X_{i} \in \mathbb{C}$, for $i=1,2, \ldots, k$.

Each transmitter is subject to an average power constraint $\mathbb{E}\left[\left|X_{i}\right|^{2}\right] \leq P_{i}, i=0,1, \ldots, k$, and the noise $Z_{i} \sim \mathcal{C N}\left(0, N_{0}\right)$ is i.i.d. over time. In the following, we define the received signal-to-noise and interference-to-noise ratios (SNR and INR, respectively):

$$
\begin{array}{lr}
\text { Network }(a) \text { of Fig. 1: } & \text { Network (b) of Fig. 1: } \\
\operatorname{SNR}_{i}:=\frac{\left|h_{i i}\right|^{2} P_{i}}{N_{0}} & \operatorname{SNR}_{i}:=\frac{\left|h_{i i}\right|^{2} P_{i}}{N_{0}} \\
\operatorname{INR}_{i}:=\frac{\left|h_{0 i}\right|^{2} P_{0}}{N_{0}} & \operatorname{INR}_{i}:=\frac{\left|h_{i 0}\right|^{2} P_{i}}{N_{0}}
\end{array}
$$

Each user $i=0,1, \ldots, k$ communicates a message $m_{i} \in\left\{1,2, \ldots, 2^{n R_{i}}\right\}$ with a codeword $\mathbf{X}_{i}^{n}:=\left(X_{i}(j)\right)_{j=1}^{n}$ drawn from a codebook $\mathcal{C}(n, i)$ the codewords of which satisfy the 


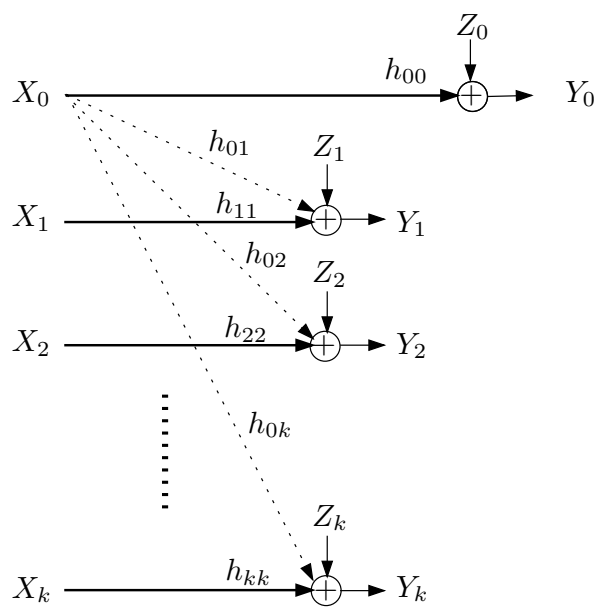

(a)

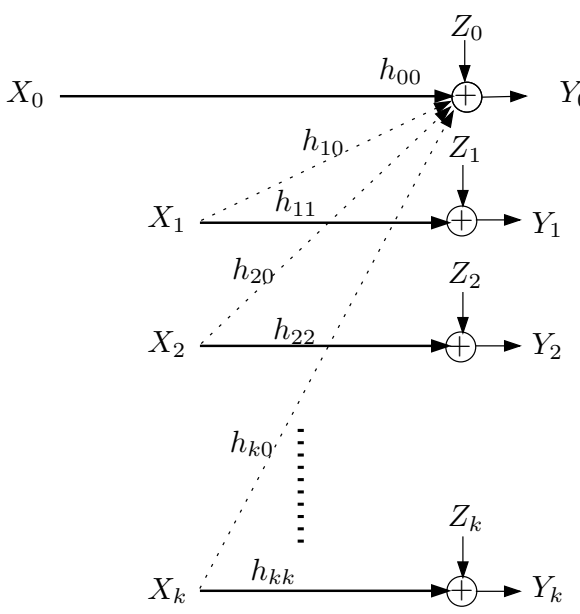

(b)

Figure 2: The many-receiver, single-interferer network in (a) and the single-receiver, many-interferer network in (b). The long range user channel input is $X_{0}$ and output $Y_{0}$.

average transmit power constraint

$$
\sum_{j=1}^{n}\left|X_{i}(j)\right|^{2} \leq n P_{i}, \quad i=1,2, \ldots, k
$$

Throughout this paper, we assume that $P_{i}=P$ for $i=0,1, \ldots, k$ and define the transmit SNR by ${ }^{1}$

$$
\mathrm{SNR}:=\frac{P}{N_{0}}
$$

\footnotetext{
${ }^{1}$ The differences in the power-profiles of the transmitters can be expressed through the channel gains to their receivers.
} 
At each receiver, the decoding function $D_{i}^{n}: \mathbb{C}^{n} \mapsto\left\{1,2, \ldots, 2^{n R_{i}}\right\}$ produces an estimate $\hat{m}_{i}$ of the transmitted message $m_{i}$ of its transmitter and error occurs if $\hat{m}_{i} \neq m_{i}$. The average error probability for user $i$ is given by

$$
\epsilon_{i, n}:=\mathbb{E}\left[\mathbb{P}\left(\hat{m}_{i} \neq m_{i}\right)\right],
$$

where the expectation is taken over the uniform and independent distribution of the messages $m_{0}, m_{1}, \ldots, m_{k}$. A rate tuple $\left(R_{0}, R_{1}, \ldots, R_{k}\right)$ is achievable if there exists a family of codebook tuples $\{(\mathcal{C}(n, 1), \mathcal{C}(n, 2), \ldots, \mathcal{C}(n, k))\}_{i=1}^{n}$ for which the error probabilities $\epsilon_{i, n}$, $i=0,1, \ldots, k$ go to zero as $n \rightarrow \infty$.

\subsection{Approximating the capacity region}

We first introduce definitions of two approximations to the capacity region, which we will use to state our main results.

Definition 2.1 An achievable rate region is said to be within one bit of the capacity region of a given network if, for any rate tuple $\left(R_{0}, R_{1}, R_{2}, \ldots, R_{k}\right)$ on the boundary of the achievable region, the rate tuple $\left(R_{0}, R_{1}+1, R_{2}+1, \ldots, R_{k}+1\right)$ is not achievable.

We note that this definition of "within one bit" is stronger than the one in [4] since it quantifies the gap from capacity in the direction of the $i$-th coordinate to be at most one bit for every choice of $R_{0}$.

Let $\mathcal{C}$ denote the capacity region of a network of type $(a)$ or type $(b)$ in Fig. 1 . Let $\widetilde{\mathcal{D}}$ be given by

$$
\widetilde{\mathcal{D}}:=\left\{\left(\frac{R_{i}}{\log \mathrm{SNR}}\right)_{i=1}^{k}:\left(R_{i}\right)_{i=1}^{k} \in \mathcal{C}\right\} .
$$

The following definition is essentially identical to the one introduced in [4].

Definition 2.2 The degree-of-freedom region is defined to be

$$
\mathcal{D}:=\lim \widetilde{\mathcal{D}}
$$

where the limit is taken as $\mathrm{SNR}, \mathrm{SNR}_{i}, \mathrm{INR}_{i} \rightarrow \infty$, while keeping fixed the ratios

$$
C_{i}:=\frac{\log \mathrm{SNR}_{i}}{\log \mathrm{SNR}}, \quad \text { and } \quad I_{i}:=\frac{\log \mathrm{INR}_{i}}{\log \mathrm{SNR}}, \quad i=0,1, \ldots, k .
$$

\section{The Main Results}

In the next three theorems, we state the main results of our paper. 
Theorem 3.1 Suppose that $I \mathrm{INR}_{1} \geq \mathrm{INR} 2 \geq, \ldots, \geq \mathrm{INR}_{k}$. The degree-of-freedom region of the network of Fig. 2 (a) is given by

$$
\begin{aligned}
d_{i} \leq & C_{i}, \\
d_{0}+\sum_{i \in \mathcal{S}} R_{i} \leq & \max \left\{I_{j_{l}}, C_{j_{l}}\right\}+\max \left\{I_{j_{l-1}}-I_{j_{l}}, C_{j_{l-1}}\right\}+ \\
\cdots+\max \left\{I_{j_{1}}-I_{j_{2}}, C_{j_{1}}\right\}+\left(C_{0}-I_{j_{1}}\right)^{+}, &
\end{aligned}
$$

for any set $\mathcal{S}=\left\{j_{1}, j_{2}, \cdots, j_{l}\right\} \subset\{1,2,3, \cdots, k\}$ with $j_{1}<j_{2}<\cdots<j_{l}$.

The scheme that achieves the degree-of-freedom region of the network is multilevel superposition coding, performed by the long-range transmitter. This can be thought of a generalization of the scheme presented in [4] (and based on [6]), for the two-user channel. Intuitively, by appropriately choosing the powers for the different components of the superposition codeword, the received power of each of the undecodable codeword components at a given short-range receiver can be forced to be below the noise floor. In other words, every codeword component that arrives above the noise floor at a given receiver is decoded and cancelled-off. The degree of freedom region can also be achieved using a power control strategy without multilevel superposition coding, for the case when $\mathrm{SNR}_{i} \geq \mathrm{INR}_{i}$, for $i=1,2, \ldots, k$.

Theorem 3.2 Suppose that $\mathrm{SNR}_{0} \geq \mathrm{INR}_{i}$ and $\mathrm{SNR}_{i} \geq \mathrm{INR}_{i}$ for $i=1,2, \ldots, k$ and consider the network shown in Fig. 2 (a). Then, the union over $\gamma \in[0,1]$ of the rate regions defined by

$$
\begin{aligned}
R_{0} & \leq \log \left(1+\gamma \mathrm{SNR}_{0}\right) \\
R_{i} & \leq \log \left(1+\frac{\mathrm{SNR}_{i}}{1+\gamma \mathrm{INR}_{i}}\right)
\end{aligned}
$$

for $i=1,2, \ldots, k$, is within one bit of the capacity region of the network.

The scheme used to obtain this region is a power control policy: the long-range transmitter reduces its transmit SNR from SNR to $\gamma$ SNR, and each of the short-range receivers simply treats interference as noise.

Theorem 3.3 The degree-of-freedom region of the network of Fig. 2 (b) with $\mathrm{SNR}_{i} \geq$ $\mathrm{INR}_{i}$ and $\mathrm{SNR}_{0} \geq \mathrm{INR}_{i}$ for $i=1,2, \ldots, k$, is given by

$$
\begin{aligned}
d_{i} & \leq C_{i}, \\
d_{0}+d_{i} & \leq C_{0}+C_{i}-I_{i},
\end{aligned}
$$

for $i=1,2, \ldots, k$.

In this case, the degree-of-freedom-optimal scheme is for the short-range transmitters to lower their transmit power and the long-range receiver to treat all interference as noise. Depending on which point on the boundary of the degree-of-freedom region the network is operating, one of the users is favored over the others. If this user turns out to be a short-range user, this user should use full transmit power while the other short-range users lower their transmit power so that the interference they create for the long-range user is at the same level as the interference caused by the favored user. 


\section{Proofs of the Main Results}

\subsection{Proof of Theorem 3.1}

\subsubsection{The outer bound}

Throughout the paper, we will use the high-SNR approximations ${ }^{2}$

$$
\begin{aligned}
\log \left(1+\mathrm{INR}_{1}+\mathrm{SNR}_{1}\right) & \approx \max \left\{\log \mathrm{INR}_{1}, \log \mathrm{SNR}_{1}\right\} \\
\log \left(\frac{1+\mathrm{SNR}_{0}}{1+\mathrm{INR}_{1}}\right) & \approx\left(\log \mathrm{SNR}_{0}-\log \mathrm{INR}_{1}\right)^{+}
\end{aligned}
$$

We define the interference plus noise term at each receiver $i$ and time $m$ as

$$
S_{i}(m)=h_{0 i} X_{0}(m)+Z_{i}(m), \text { for } i=1,2, \cdots, k,
$$

and

$$
S_{i}^{n}=\left(S_{i}(1), S_{i}(2), \ldots, S_{i}(n)\right) \text { for } i=1,2, \cdots, k,
$$

Without loss of generality we can assume $\mathcal{S}=\{1,2,3, \cdots, k\}$, i.e., we can characterize the bound on $d_{0}+d_{1}+\ldots, d_{n}$. We begin by deriving an upper bound on $R_{0}+R_{1}+\cdots R_{k}$ (for ease of notation, we omit the $n \epsilon_{n}$ terms coming from Fano's inequality).

$$
\begin{aligned}
n & \left(R_{0}+R_{1}+\cdots R_{k}\right) \\
\leq & I\left(X_{0}^{n} ; Y_{0}^{n}\right)+I\left(X_{1}^{n} ; Y_{1}^{n}\right)+\cdots+I\left(X_{k-1}^{n} ; Y_{k-1}^{n}\right)+I\left(X_{k}^{n} ; Y_{k}^{n}\right) \\
\leq & I\left(X_{0}^{n} ; Y_{0}^{n}, S_{1}^{n}, S_{2}^{n}, \cdots, S_{k}^{n}\right)+I\left(X_{1}^{n} ; Y_{1}^{n}, S_{2}^{n}, S_{3}^{n}, \cdots, S_{k}^{n}\right)+\cdots+I\left(X_{k-1}^{n} ; Y_{k-1}^{n}, S_{k}^{n}\right)+I\left(X_{k}^{n} ; Y_{k}^{n}\right) \\
= & I\left(X_{0}^{n} ; S_{k}^{n}\right)+I\left(X_{0}^{n} ; S_{k-1}^{n} \mid S_{k}^{n}\right)+\cdots+I\left(X_{0}^{n} ; S_{1}^{n} \mid S_{2}^{n}, \cdots, S_{k}^{n}\right)+I\left(X_{0}^{n} ; Y_{0}^{n} \mid S_{1}^{n}, \cdots, S_{k}^{n}\right) \\
& +I\left(X_{1}^{n} ; Y_{1}^{n} \mid S_{2}^{n}, \cdots, S_{k}^{n}\right)+\cdots+I\left(X_{k-1}^{n} ; Y_{k-1}^{n} \mid S_{k}^{n}\right)+I\left(X_{k}^{n} ; Y_{k}^{n}\right) \\
= & h\left(S_{k}^{n}\right)-h\left(Z_{k}^{n}\right)+h\left(S_{k-1}^{n} \mid S_{k}^{n}\right)-h\left(Z_{k-1}^{n}\right)+\cdots+h\left(S_{1}^{n} \mid S_{2}^{n}, \cdots, S_{k}^{n}\right)-h\left(Z_{1}^{n}\right)+ \\
& h\left(Y_{0}^{n} \mid S_{1}^{n}, \cdots, S_{k}^{n}\right)-h\left(Z_{0}^{n}\right)+h\left(Y_{1}^{n} \mid S_{2}^{n}, \cdots, S_{k}^{n}\right)-h\left(Y_{1}^{n} \mid X_{1}^{n}, S_{2}^{n}, \cdots, S_{k}^{n}\right)+\cdots \\
& +h\left(Y_{k-1}^{n} \mid S_{k}^{n}\right)-h\left(Y_{k-1}^{n} \mid X_{k-1}^{n} S_{k}^{n}\right)+h\left(Y_{k}^{n}\right)-h\left(S_{k}^{n}\right) \\
= & h\left(S_{k}^{n}\right)-h\left(Z_{k}^{n}\right)+h\left(S_{k-1}^{n} \mid S_{k}^{n}\right)-h\left(Z_{k-1}^{n}\right)+\cdots+h\left(S_{1}^{n} \mid S_{2}^{n}, \cdots, S_{k}^{n}\right)-h\left(Z_{1}^{n}\right) \\
& +h\left(Y_{0}^{n} \mid S_{1}^{n}, \cdots, S_{k}^{n}\right)-h\left(Z_{0}^{n}\right)+h\left(Y_{1}^{n} \mid S_{2}^{n}, \cdots, S_{k}^{n}\right)-h\left(S_{1}^{n} \mid S_{2}^{n}, \cdots, S_{k}^{n}\right)+\cdots+h\left(Y_{k-1}^{n} \mid S_{k}^{n}\right) \\
& -h\left(S_{k-1}^{n} \mid S_{k}^{n}\right)+h\left(Y_{k}^{n}\right)-h\left(S_{k}^{n}\right) \\
= & h\left(Y_{0}^{n} \mid S_{1}^{n}, \cdots, S_{k}^{n}\right)+h\left(Y_{1}^{n} \mid S_{2}^{n}, \cdots, S_{k}^{n}\right)+\cdots+h\left(Y_{k-1}^{n} \mid S_{k}^{n}\right)+h\left(Y_{k}^{n}\right)-h\left(Z_{k}^{n}\right)-\cdots-h\left(Z_{0}^{n}\right) \\
\leq & h\left(Y_{0}^{n} \mid S_{1}^{n}\right)+h\left(Y_{1}^{n} \mid S_{2}^{n}\right)+\cdots+h\left(Y_{k-1}^{n} \mid S_{k}^{n}\right)+h\left(Y_{k}^{n}\right)-h\left(Z_{k}^{n}\right)-\cdots-h\left(Z_{0}^{n}\right) \\
\leq & \sum_{i=1}^{n}\left[h\left(Y_{0, i} \mid S_{1, i}\right)+h\left(Y_{1, i} \mid S_{2, i}\right)+\cdots+h\left(Y_{k-1, i} \mid S_{k, i}\right)+h\left(Y_{k, i}\right)\right]-h\left(Z_{k}^{n}\right)-\cdots-h\left(Z_{0}^{n}\right) .
\end{aligned}
$$

We observe that Gaussian inputs maximize the conditional and unconditional entropies in the above expression. Evaluating the bound with Gaussian inputs, using convexity of

\footnotetext{
${ }^{2}$ which satisfy the property that the higher order terms are $O(1)$, i.e., the approximation error vanishes as $\mathrm{SNR}, \mathrm{INR}_{1}, \mathrm{SNR}_{0}, \mathrm{SNR}_{1} \rightarrow \infty$.
} 
$\log$ function and the power constraint, dividing by $n$ and letting $n \rightarrow \infty$ and applying the high-SNR approximations, we obtain the following bound on the degrees of freedom:

$$
\begin{aligned}
d_{0}+d_{1}+\ldots+d_{k} \leq & \left(C_{0}-I_{1}\right)^{+}+\max \left\{I_{1}-I_{2}, C_{1}\right\}+\cdots \\
& +\max \left\{I_{k-1}-I_{k-2}, C_{k-1}\right\}+\max \left\{I_{k}, C_{k}\right\} .
\end{aligned}
$$

The individual rate bounds in the statement of Theorem 3.1 follow trivially from Fano's inequality.

\subsubsection{The inner bound}

We use a multilevel superposition coding scheme as described in the following. Suppose that $I_{N R} \geq I N R_{2} \geq, \ldots, \geq I N R_{k}$. User 0 splits its message $m_{0}$ into $k+1$ independent messages $u, w, w_{1}, w_{2}, \ldots, w_{k-1}$ and generates corresponding i.i.d. Gaussian codewords $X_{u}^{n}, X_{w}^{n}, X_{w_{1}}^{n}, \ldots, X_{w_{k-1}}^{n}$ of average power

$$
\begin{aligned}
P_{u} & :=\frac{\mathrm{SNR}}{\mathrm{INR}_{1}} \\
P_{w} & :=\mathrm{SNR}, \\
P_{w_{i}} & :=\frac{\mathrm{SNR}}{\mathrm{INR}_{i+1}}, \quad i=1,2, \ldots, k-1 .
\end{aligned}
$$

The transmitted codeword is then given by the superposition of the individual ones:

$$
X_{0}^{n}=X_{w}^{n}+X_{u}^{n}+X_{w_{1}}^{n}+\ldots+X_{w_{k-1}}^{n} .
$$

Message $u$ is private and is decoded only by receiver 0 and message $w$ is public and is decoded by all of the receivers. Message $w_{1}$ is decoded only by receivers 0 and 1 ; message $w_{2}$ is decoded by receivers 0,1 , and 2 ; message $w_{3}$ is decoded by receivers $0,1,2$, and 3 ; and the last message $w_{k-1}$ is decoded by all but receiver $k$. Next we use induction to prove that this scheme can acieve the degree of freedom region given in Theorem 3.1.

Step 1: $k=2$ (3-node network)

For each of the independent messages $u, w, w_{1}$ of user 0 and messages $m_{1}, m_{2}$ of users 1 and 2 , we associate a degree of freedom $d_{u}, d_{w}, d_{w_{1}}$, and $d_{1}, d_{2}$, respectively. Each of the receiver can be thought of as the receiver of a MAC channel. Receiver 0 needs to decode $u, w, w_{1}$, receiver 1 needs to decode $w, w_{1}, m_{1}$ and receiver 2 needs to decode $w, m_{2}$. The achievable degree-of-freedom region is given by the following set of inequalities: 


$$
\begin{aligned}
& \text { @ Receiver } 0 @ \text { @ Receiver 1@ @ Receiver } 2 \\
& d_{w} \leq C_{0}, \quad d_{w} \leq I_{1}, \quad d_{w} \leq I_{2}, \\
& d_{w_{1}} \leq\left(C_{0}-I_{2}\right)^{+}, \quad d_{w_{1}} \leq I_{1}-I_{2}, \quad d_{2} \leq C_{2}, \\
& d_{u} \leq\left(C_{0}-I_{1}\right)^{+}, \quad d_{1} \leq C_{1}, \quad d_{w}+d_{2} \leq \max \left\{I_{2}, C_{2}\right\}, \\
& d_{w}+d_{w_{1}} \leq C_{0}, \quad d_{w}+d_{w_{1}} \leq I_{1}, \\
& d_{w}+d_{u} \leq C_{0}, \quad d_{w}+d_{1} \leq \max \left\{I_{1}, C_{1}\right\}, \\
& d_{w_{1}}+d_{u} \leq\left(C_{0}-I_{2}\right)^{+}, \quad d_{w_{1}}+d_{1} \leq \max \left\{I_{1}-I_{2}, C_{1}\right\}, \\
& d_{w}+d_{w_{1}}+d_{u} \leq C_{0}, \quad d_{w}+d_{w_{1}}+d_{1} \leq \max \left\{I_{1}, C_{1}\right\} .
\end{aligned}
$$

By removing the redundant inequalities, we obtain the following region: At receiver 0:

$$
\begin{aligned}
d_{w}+d_{w_{1}}+d_{u} & \leq C_{0}, \\
d_{w_{1}}+d_{u} & \leq\left(C_{0}-I_{2}\right)^{+}, \\
d_{u} & \leq\left(C_{0}-I_{1}\right)^{+} .
\end{aligned}
$$

At receiver 1:

$$
\begin{aligned}
d_{w}+d_{w_{1}} & \leq I_{1} \\
d_{w_{1}} & \leq I_{1}-I_{2} \\
d_{1}+d_{w}+d_{w_{1}} & \leq \max \left\{I_{1}, C_{1}\right\} \\
d_{1}+d_{w_{1}} & \leq \max \left\{I_{1}-I_{2}, C_{1}\right\} \\
d_{1} & \leq C_{1}
\end{aligned}
$$

At receiver 2:

$$
\begin{aligned}
d_{w} & \leq I_{2}, \\
d_{2}+d_{w} & \leq \max \left\{I_{2}, C_{2}\right\}, \\
d_{2} & \leq C_{2} .
\end{aligned}
$$

We can use Fourier-Motzkin elimination to get an achievable region in terms of $d_{0}, d_{1}$ and $d_{2}$. To do that, note that $d_{0}=d_{w}+d_{w_{1}}+d_{u}$ and all the rates are non-negative, we add the following obvious bounds:

$$
\begin{aligned}
-d_{0}+d_{w}+d_{w_{1}}+d_{u} & \leq 0 \\
d_{0}-d_{w}-d_{w_{1}}-d_{u} & \leq 0 \\
-d_{u} & \leq 0 \\
-d_{w_{1}} & \leq 0 \\
-d_{w} & \leq 0
\end{aligned}
$$


Note that $d_{u}$ only appears in (6) and (9). We can eliminate $d_{u}$ by adding each of the inequalities in which the coefficient of $d_{u}$ is +1 to each of the inequalities in which the coefficient of $d_{u}$ is -1 . After doing that, we have the bounds in (7), (8) and the following.

$$
\begin{aligned}
d_{0} & \leq C_{0} \\
d_{0}-d_{w} & \leq\left(C_{0}-I_{2}\right)^{+}, \\
d_{0}-d_{w}-d_{w_{1}} & \leq\left(C_{0}-I_{1}\right)^{+} \\
d_{w}+d_{w_{1}} & \leq C_{0} \\
d_{w_{1}} & \leq\left(C_{0}-I_{2}\right)^{+}, \\
-d_{0}+d_{w}+d_{w_{1}} & \leq 0 \\
-d_{w_{1}} & \leq 0 \\
-d_{w} & \leq 0
\end{aligned}
$$

We then eliminate $d_{w_{1}}$ from (7) and (10). After doing that, we have the bounds in (8) and the following.

$$
\begin{aligned}
d_{0} & \leq C_{0} \\
d_{0}-d_{w} & \leq\left(C_{0}-I_{2}\right)^{+} \\
d_{0} & \leq\left(C_{0}-I_{1}\right)^{+}+I_{1}, \\
d_{0}-d_{w} & \leq\left(C_{0}-I_{1}\right)^{+}+I_{1}-I_{2}, \\
d_{0}+d_{1} & \leq\left(C_{0}-I_{1}\right)^{+}+\max \left\{I_{1}, C_{1}\right\}, \\
d_{0}+d_{1}-d_{w} & \leq\left(C_{0}-I_{1}\right)^{+}+\max \left\{I_{1}-I_{2}, C_{1}\right\}, \\
d_{0} & \leq\left(C_{0}-I_{1}\right)^{+}+C_{0}, \\
d_{0}-d_{w} & \leq\left(C_{0}-I_{1}\right)^{+}+\left(C_{0}-I_{2}\right)^{+}, \\
d_{w} & \leq I_{1} \\
d_{1}+d_{w} & \leq \max \left\{I_{1}, C_{1}\right\} \\
d_{1} & \leq \max \left\{I_{1}-I_{2}, C_{1}\right\} \\
d_{w} & \leq C_{0}, \\
-d_{0}+d_{w} \leq 0 & \\
d_{1} \leq C_{1} & \\
-d_{w} & \leq 0 .
\end{aligned}
$$


After removing the redundant bounds in the above equation we get

$$
\begin{aligned}
d_{0} & \leq C_{0}, \\
d_{0}-d_{w} & \leq\left(C_{0}-I_{2}\right)^{+}, \\
d_{0}+d_{1} & \leq\left(C_{0}-I_{1}\right)^{+}+\max \left\{I_{1}, C_{1}\right\}, \\
d_{0}+d_{1}-d_{w} & \leq\left(C_{0}-I_{1}\right)^{+}+\max \left\{I_{1}-I_{2}, C_{1}\right\}, \\
d_{w} & \leq I_{1}, \\
d_{1}+d_{w} & \leq \max \left\{I_{1}, C_{1}\right\}, \\
d_{w} & \leq C_{0}, \\
-d_{0}+d_{w} \leq 0 & \\
d_{1} \leq C_{1} & \\
-d_{w} & \leq 0 .
\end{aligned}
$$

We finally eliminate $d_{w}$ from (7) and (12). After doing that, we have the following bounds.

$$
\begin{aligned}
d_{0} & \leq C_{0}, \\
d_{0} & \leq\left(C_{0}-I_{2}\right)^{+}+I_{2}, \\
d_{0}+d_{2} & \leq\left(C_{0}-I_{2}\right)^{+}+\max \left\{I_{2}, C_{2}\right\}, \\
d_{0} & \leq\left(C_{0}-I_{2}\right)^{+}+I_{1}, \\
d_{0}+d_{1} & \leq\left(C_{0}-I_{2}\right)^{+}+\max \left\{I_{1}, C_{1}\right\}, \\
d_{0} & \leq\left(C_{0}-I_{2}\right)^{+}+C_{0}, \\
d_{0}+d_{1} & \leq\left(C_{0}-I_{1}\right)^{+}+\max \left\{I_{1}, C_{1}\right\}, \\
d_{0}+d_{1} & \leq\left(C_{0}-I_{1}\right)^{+}+\max \left\{I_{1}-I_{2}, C_{1}\right\}+I_{2}, \\
d_{0}+d_{1}+d_{2} & \leq\left(C_{0}-I_{1}\right)^{+}+\max \left\{I_{1}-I_{2}, C_{1}\right\}+\max \left\{I_{2}, C_{2}\right\}, \\
d_{0}+d_{1} & \leq\left(C_{0}-I_{1}\right)^{+}+\max \left\{I_{1}-I_{2}, C_{1}\right\}+I_{1}, \\
d_{0}+2 d_{1} & \leq\left(C_{0}-I_{1}\right)^{+}+\max \left\{I_{1}-I_{2}, C_{1}\right\}+\max \left\{I_{1}, C_{1}\right\}, \\
d_{0}+d_{1} & \leq\left(C_{0}-I_{1}\right)^{+}+\max \left\{I_{1}-I_{2}, C_{1}\right\}+C_{0}, \\
d_{1} & \leq\left(C_{0}-I_{1}\right)^{+}+\max \left\{I_{1}-I_{2}, C_{1}\right\}, \\
d_{2} & \leq \max \left\{I_{2}, C_{2}\right\}, \\
d_{1} & \leq \max \left\{I_{1}, C_{1}\right\}, \\
d_{2} & \leq C_{2}, \\
d_{1} & \leq C_{1} .
\end{aligned}
$$

After removing the redundant bounds in the above equation we get

$$
\begin{aligned}
d_{0} & \leq C_{0}, \\
d_{1} & \leq C_{1}, \\
d_{2} & \leq C_{2}, \\
d_{0}+d_{1} & \leq \max \left\{I_{1}, C_{1}\right\}+\left(C_{0}-I_{1}\right)^{+}, \\
d_{0}+d_{2} & \leq \max \left\{I_{2}, C_{2}\right\}+\left(C_{0}-I_{2}\right)^{+}, \\
d_{0}+d_{1}+d_{2} & \leq\left(C_{0}-I_{1}\right)^{+}+\max \left\{I_{1}-I_{2}, C_{1}\right\}+\max \left\{I_{2}, C_{2}\right\} .
\end{aligned}
$$


Step 2: $(k+1)$-node network.

We have

$$
x_{0}=x_{w}+x_{w_{k-1}}+x_{w_{k-2}}+\cdots+x_{w_{1}}+x_{u} .
$$

If we examine all the constraints for $(k+1)$-node network, we have the following non-trivial constraints at each receiver:

At receiver 0:

$$
\begin{aligned}
d_{w}+d_{w_{k-1}}+d_{w_{k-2}}+\cdots+d_{w_{1}}+d_{u} & \leq C_{0}, \\
d_{w_{k-1}}+d_{w_{k-2}}+\cdots+d_{w_{1}}+d_{u} & \leq\left(C_{0}-I_{k}\right)^{+}, \\
d_{w_{k-2}}+\cdots+d_{w_{1}}+d_{u} & \leq\left(C_{0}-I_{k-1}\right)^{+}, \\
\vdots & \\
d_{w_{1}}+d_{u} & \leq\left(C_{0}-I_{2}\right)^{+}, \\
d_{u} & \leq\left(C_{0}-I_{1}\right)^{+} .
\end{aligned}
$$

At receiver 1:

$$
\begin{aligned}
d_{w}+d_{w_{k-1}}+d_{w_{k-2}}+\cdots+d_{w_{1}} & \leq I_{1} \\
d_{w_{k-1}}+d_{w_{k-2}}+\cdots+d_{w_{1}} & \leq I_{1}-I_{k}, \\
d_{w_{k-2}}+\cdots+d_{w_{1}} & \leq I_{1}-I_{k-1}, \\
\vdots & \\
d_{w_{2}}+d_{w_{1}} & \leq I_{1}-I_{3}, \\
d_{w_{1}} & \leq I_{1}-I_{2}, \\
d_{1}+d_{w}+d_{w_{k-1}}+d_{w_{k-2}}+\cdots+d_{w_{1}} & \leq \max \left\{I_{1}, C_{1}\right\}, \\
d_{1}+d_{w_{k-1}}+d_{w_{k-2}}+\cdots+d_{w_{1}} & \leq \max \left\{I_{1}-I_{k}, C_{1}\right\}, \\
d_{1}+d_{w_{k-2}}+\cdots+d_{w_{1}} & \leq \max \left\{I_{1}-I_{k-1}, C_{1}\right\}, \\
& \vdots \\
d_{1}+d_{w_{2}}+d_{w_{1}} & \leq \max \left\{I_{1}-I_{3}, C_{1}\right\}, \\
d_{1}+d_{w_{1}} & \leq \max \left\{I_{1}-I_{2}, C_{1}\right\}, \\
d_{1} & \leq C_{1} .
\end{aligned}
$$


At receiver 2:

$$
\begin{aligned}
d_{w}+d_{w_{k-1}}+d_{w_{k-2}}+\cdots+d_{w_{2}} & \leq I_{2} \\
d_{w_{k-1}}+d_{w_{k-2}}+\cdots+d_{w_{2}} & \leq I_{2}-I_{k}, \\
d_{w_{k-2}}+\cdots+d_{w_{2}} & \leq I_{2}-I_{k-1}, \\
\vdots & \\
d_{w_{3}}+d_{w_{2}} & \leq I_{2}-I_{4}, \\
d_{w_{2}} & \leq I_{2}-I_{3}, \\
d_{2}+d_{w}+d_{w_{k-1}}+d_{w_{k-2}}+\cdots+d_{w_{2}} & \leq \max \left\{I_{2}, C_{2}\right\}, \\
d_{2}+d_{w_{k-1}}+d_{w_{k-2}}+\cdots+d_{w_{2}} & \leq \max \left\{I_{2}-I_{k}, C_{2}\right\}, \\
d_{2}+d_{w_{k-2}}+\cdots+d_{w_{2}} & \leq \max \left\{I_{2}-I_{k-1}, C_{2}\right\}, \\
& \vdots \\
d_{2}+d_{w_{3}}+d_{w_{2}} & \leq \max \left\{I_{2}-I_{4}, C_{2}\right\}, \\
d_{2}+d_{w_{2}} & \leq \max \left\{I_{2}-I_{3}, C_{2}\right\}, \\
d_{2} & \leq C_{2}
\end{aligned}
$$

At receiver $k$ :

$$
\begin{aligned}
d_{w} & \leq I_{k}, \\
d_{k}+d_{w} & \leq \max \left\{I_{k}, C_{k}\right\}, \\
d_{k} & \leq C_{k} .
\end{aligned}
$$

We also add the following obvious bounds:

$$
\begin{aligned}
-d_{0}+d_{w}+d_{w_{k-1}}+d_{w_{k-2}}+\cdots+d_{w_{1}}+d_{u} & \leq 0 \\
d_{0}-d_{w}-d_{w_{k-1}}-d_{w_{k-2}}-\cdots-d_{w_{1}}-d_{u} & \leq 0 \\
-d_{u} & \leq 0 \\
-d_{w_{1}} & \leq 0 \\
\vdots & \\
-d_{w} & \leq 0
\end{aligned}
$$

We assume that we get the degree of freedom region as described in Theorem 3.1 for $(k+1)$-node network after performing FM elimination. 
In particular, after eliminate $d_{u}$ from (14) and (18), we have the following bounds

$$
\begin{aligned}
d_{0} & \leq C_{0} \\
d_{0}-d_{w} & \leq\left(C_{0}-I_{k}\right)^{+}, \\
d_{0}-d_{w}-d_{w_{k-1}} & \leq\left(C_{0}-I_{k-1}\right)^{+}, \\
\vdots & \\
d_{0}-d_{w}-d_{w_{k-1}}-\cdots-d_{w_{2}} & \leq\left(C_{0}-I_{2}\right)^{+}, \\
d_{0}-d_{w}-d_{w_{k-1}}-\cdots-d_{w_{2}}-d_{w_{1}} & \leq\left(C_{0}-I_{1}\right)^{+}, \\
d_{w}+d_{w_{k-1}}+\cdots+d_{w_{1}} & \leq C_{0} \\
d_{w_{k-1}}+d_{w_{k-2}}+\cdots+d_{w_{1}} & \leq\left(C_{0}-I_{k}\right)^{+}, \\
& \vdots \\
d_{w_{1}} & \leq\left(C_{0}-I_{2}\right)^{+}, \\
-d_{0}+d_{w}+d_{w_{k-1}}+\cdots+d_{w_{1}} & \leq 0 \\
-d_{w_{1}} & \leq 0 \\
\vdots & \\
-d_{w} & \leq 0
\end{aligned}
$$

Step 3: $(k+2)$-node network.

We have

$$
x_{0}=x_{w}+x_{w_{k}}+x_{w_{k-1}}+x_{w_{k-2}}+\cdots+x_{w_{1}}+x_{u} .
$$

If we examine all the constraints for $(\mathrm{k}+2)$-node network, we have the following nontrivial constraints at each receiver:

At receiver 0:

$$
\begin{aligned}
d_{w}+d_{w_{k}}+d_{w_{k-1}}+\cdots+d_{w_{1}}+d_{u} & \leq C_{0} \\
d_{w_{k}}+d_{w_{k-1}}+d_{w_{k-2}}+\cdots+d_{w_{1}}+d_{u} & \leq\left(C_{0}-I_{k+1}\right)^{+}, \\
d_{w_{k-1}}+d_{w_{k-2}}+\cdots+d_{w_{1}}+d_{u} & \leq\left(C_{0}-I_{k}\right)^{+}, \\
d_{w_{k-2}}+\cdots+d_{w_{1}}+d_{u} & \leq\left(C_{0}-I_{k-1}\right)^{+}, \\
\vdots & \\
d_{w_{1}}+d_{u} & \leq\left(C_{0}-I_{2}\right)^{+}, \\
d_{u} & \leq\left(C_{0}-I_{1}\right)^{+} .
\end{aligned}
$$


At receiver 1:

$$
\begin{aligned}
d_{w}+d_{w_{k}}+d_{w_{k-1}}+d_{w_{k-2}}+\cdots+d_{w_{1}} & \leq I_{1}, \\
d_{w_{k}}+d_{w_{k-1}}+d_{w_{k-2}}+\cdots+d_{w_{1}} & \leq I_{1}-I_{k+1}, \\
d_{w_{k-1}}+d_{w_{k-2}}+\cdots+d_{w_{1}} & \leq I_{1}-I_{k}, \\
d_{w_{k-2}}+\cdots+d_{w_{1}} & \leq I_{1}-I_{k-1}, \\
\vdots & \\
d_{w_{2}}+d_{w_{1}} & \leq I_{1}-I_{3}, \\
d_{w_{1}} & \leq I_{1}-I_{2}, \\
d_{1}+d_{w}+d_{w_{k}}+d_{w_{k-1}}+d_{w_{k-2}}+\cdots+d_{w_{1}} & \leq \max \left\{I_{1}, C_{1}\right\}, \\
d_{1}+d_{w_{k}}+d_{w_{k-1}}+d_{w_{k-2}}+\cdots+d_{w_{1}} & \leq \max \left\{I_{1}-I_{k+1}, C_{1}\right\}, \\
d_{1}+d_{w_{k-1}}+d_{w_{k-2}}+\cdots+d_{w_{1}} & \leq \max \left\{I_{1}-I_{k}, C_{1}\right\}, \\
d_{1}+d_{w_{k-2}}+\cdots+d_{w_{1}} & \leq \max \left\{I_{1}-I_{k-1}, C_{1}\right\}, \\
& \vdots \\
d_{1}+d_{w_{2}}+d_{w_{1}} & \leq \max \left\{I_{1}-I_{3}, C_{1}\right\}, \\
d_{1}+d_{w_{1}} & \leq \max \left\{I_{1}-I_{2}, C_{1}\right\}, \\
d_{1} & \leq C_{1} .
\end{aligned}
$$

At receiver 2:

$$
\begin{aligned}
d_{w}+d_{w_{k}}+d_{w_{k-1}}+d_{w_{k-2}}+\cdots+d_{w_{2}} & \leq I_{2}, \\
d_{w_{k}}+d_{w_{k-1}}+d_{w_{k-2}}+\cdots+d_{w_{2}} & \leq I_{2}-I_{k+1}, \\
d_{w_{k-1}}+d_{w_{k-2}}+\cdots+d_{w_{2}} & \leq I_{2}-I_{k}, \\
d_{w_{k-2}}+\cdots+d_{w_{2}} & \leq I_{2}-I_{k-1}, \\
\vdots & \\
d_{w_{3}}+d_{w_{2}} & \leq I_{2}-I_{4}, \\
d_{w_{2}} & \leq I_{2}-I_{3}, \\
d_{2}+d_{w}+d_{w_{k}}+d_{w_{k-1}}+d_{w_{k-2}}+\cdots+d_{w_{2}} & \leq \max \left\{I_{2}, C_{2}\right\}, \\
d_{2}+d_{w_{k}}+d_{w_{k-1}}+d_{w_{k-2}}+\cdots+d_{w_{2}} & \leq \max \left\{I_{2}-I_{k+1}, C_{2}\right\}, \\
d_{2}+d_{w_{k-1}}+d_{w_{k-2}}+\cdots+d_{w_{2}} & \leq \max \left\{I_{2}-I_{k}, C_{2}\right\}, \\
d_{2}+d_{w_{k-2}}+\cdots+d_{w_{2}} & \leq \max \left\{I_{2}-I_{k-1}, C_{2}\right\}, \\
& \vdots \\
d_{2}+d_{w_{3}}+d_{w_{2}} & \leq \max \left\{I_{2}-I_{4}, C_{2}\right\}, \\
d_{2}+d_{w_{2}} & \leq \max \left\{I_{2}-I_{3}, C_{2}\right\}, \\
d_{2} & \leq C_{2} .
\end{aligned}
$$

At receiver $k+1$ :

$$
\begin{aligned}
d_{w} & \leq I_{k} \\
d_{k+1}+d_{w} & \leq \max \left\{I_{k+1}, C_{k+1}\right\}, \\
d_{k+1} & \leq C_{k+1}
\end{aligned}
$$


We also add the following obvious bounds:

$$
\begin{array}{r}
-d_{0}+d_{w}+d_{w_{k}}+d_{w_{k-1}}+\cdots+d_{w_{1}}+d_{u} \leq 0 \\
d_{0}-d_{w}-d_{w_{k}}-d_{w_{k-1}}-\cdots-d_{w_{1}}-d_{u} \leq 0 \\
-d_{u} \leq 0 \\
-d_{w_{1}} \leq 0 \\
\vdots \\
-d_{w} \leq 0
\end{array}
$$

Comparing the bounds at receiver $l+1$ in step 3 with the bounds at receiver $l$ in step 2 for $l=1,2, \cdots, k$, we can see that if we make change of $I_{j}$ to $I_{j+1}, C_{j}$ to $C_{j+1}, d_{j}$ to $d_{j+1}$, and $d_{w_{j}}$ to $d_{w_{j+1}}$ for $j=1,2, \cdots, k$ in the bounds at receiver $l$ in step 2 , we get exactly the bounds at receiver $l+1$ in step 3 . The idea is to show that the bounds we get after FM elimination of $d_{u}$ and $d_{w_{1}}$ in step 3 have a similar relation with the bounds (19) that we get after FM elimination of $d_{u}$ in step 2.

After eliminating $d_{u}$ and $d_{w_{1}}$ from (20), (21), (24), and getting rid of redundant bounds, we have the following bounds:

$$
\begin{aligned}
d_{1} & \leq C_{1}, \\
d_{0} & \leq C_{0}, \\
d_{0}-d_{w} & \leq\left(C_{0}-I_{k+1}\right)^{+}, \\
d_{0}-d_{w}-d_{w_{k}} & \leq\left(C_{0}-I_{k}\right)^{+}, \\
\vdots & \\
d_{0}-d_{w}-d_{w_{k}}-\cdots-d_{w_{3}}-d_{w_{2}} & \leq\left(C_{0}-I_{3}\right)^{+}, \\
d_{0}-d_{w}-d_{w_{k}}-\cdots-d_{w_{2}} & \leq\left(C_{0}-I_{2}\right)^{+}, \\
d_{1}+d_{0} & \leq \max \left\{I_{1}, C_{1}\right\}+\left(C_{0}-I_{1}\right)^{+}, \\
d_{1}+d_{0}-d_{w} & \leq \max \left\{I_{1}-I_{k+1}, C_{1}\right\}+\left(C_{0}-I_{1}\right)^{+}, \\
d_{1}+d_{0}-d_{w}-d_{w_{k}} & \leq \max \left\{I_{1}-I_{k+1}, C_{1}\right\}+\left(C_{0}-I_{1}\right)^{+}, \\
\vdots & \\
d_{1}+d_{0}-d_{w}-d_{w_{k}}-\cdots-d_{w_{3}}-d_{w_{2}} & \leq \max \left\{I_{1}-I_{3}, C_{1}\right\}+\left(C_{0}-I_{1}\right)^{+}, \\
d_{1}+d_{0}-d_{w}-d_{w_{k}}-\cdots-d_{w_{2}} & \leq \max \left\{I_{1}-I_{2}, C_{1}\right\}+\left(C_{0}-I_{1}\right)^{+}, \\
d_{w}+d_{w_{k}}+\cdots+d_{w_{2}} & \leq C_{0}, \\
d_{w_{k}}+d_{w_{k-1}}+\cdots+d_{w_{2}} & \leq\left(C_{0}-I_{k+1}\right)^{+}, \\
\vdots & \\
d_{w_{2}} & \leq\left(C_{0}-I_{3}\right)^{+}, \\
-d_{w_{2}} & \leq 0, \\
\vdots & \\
-d_{w} & \leq 0 . \\
-d_{0}+d_{w}+d_{w_{k-1}}+\cdots+d_{w_{2}} & \leq 0 \\
-d_{1} &
\end{aligned}
$$


We can rewrite these bounds into two groups:

$$
\begin{aligned}
d_{1} & \leq C_{1}, \\
d_{0} & \leq C_{0}, \\
d_{0}-d_{w} & \leq\left(C_{0}-I_{k+1}\right)^{+}, \\
d_{0}-d_{w}-d_{w_{k}} & \leq\left(C_{0}-I_{k}\right)^{+}, \\
\vdots & \\
d_{0}-d_{w}-d_{w_{k}}-\cdots-d_{w_{3}}-d_{w_{2}} & \leq\left(C_{0}-I_{3}\right)^{+}, \\
d_{0}-d_{w}-d_{w_{k}}-\cdots-d_{w_{2}} & \leq\left(C_{0}-I_{2}\right)^{+}, \\
d_{w}+d_{w_{k}}+\cdots+d_{w_{2}} & \leq C_{0}, \\
d_{w_{k}}+d_{w_{k-1}}+\cdots+d_{w_{2}} & \leq\left(C_{0}-I_{k+1}\right)^{+}, \\
\vdots & \\
d_{w_{2}} & \leq\left(C_{0}-I_{3}\right)^{+}, \\
-d_{w_{2}} & \leq 0 \\
d_{w}+d_{w_{k-1}}+\cdots+d_{w_{2}} & \leq 0 \\
\vdots & \\
-d_{w} & \leq 0 .
\end{aligned}
$$

and

$$
\begin{aligned}
d_{1}+d_{0} & \leq \max \left\{I_{1}, C_{1}\right\}+\left(C_{0}-I_{1}\right)^{+}, \\
d_{1}+d_{0}-d_{w} & \leq \max \left\{I_{1}-I_{k+1}, C_{1}\right\}+\left(C_{0}-I_{1}\right)^{+}, \\
d_{1}+d_{0}-d_{w}-d_{w_{k}} & \leq \max \left\{I_{1}-I_{k+1}, C_{1}\right\}+\left(C_{0}-I_{1}\right)^{+}, \\
\vdots & \\
d_{1}+d_{0}-d_{w}-d_{w_{k}}-\cdots-d_{w_{3}}-d_{w_{2}} & \leq \max \left\{I_{1}-I_{3}, C_{1}\right\}+\left(C_{0}-I_{1}\right)^{+}, \\
d_{1}+d_{0}-d_{w}-d_{w_{k}}-\cdots-d_{w_{2}} & \leq \max \left\{I_{1}-I_{2}, C_{1}\right\}+\left(C_{0}-I_{1}\right)^{+}, \\
d_{w}+d_{w_{k}}+\cdots+d_{w_{2}} & \leq C_{0} \\
d_{w_{k}}+d_{w_{k-1}}+\cdots+d_{w_{2}} & \leq\left(C_{0}-I_{k+1}\right)^{+} \\
\vdots & \\
d_{w_{2}} & \leq\left(C_{0}-I_{3}\right)^{+} \\
-d_{w_{2}} & \leq 0 \\
\vdots & \\
-d_{0}+d_{w}+d_{w_{k-1}}+\cdots & \leq 0
\end{aligned}
$$

It is easy to see that the bound in terms of $d_{0}, d_{1}, d_{2}, \cdots, d_{k}$ is the union of the set of bounds derived by FM elimination using (26) with the rest of bounds at receiver 2 to receiver $k+1$ and the set of bounds derived by FM elimination by using (27) with the rest of bounds at receiver 2 to receiver $k+1$. 
Comparing the first group $((26))$ with the bounds in equation (19) in step 2, we can see that if we make change of $I_{j}$ to $I_{j+1}, C_{j}$ to $C_{j+1}, d_{j}$ to $d_{j+1}$, and $d_{w_{j}}$ to $d_{w_{j+1}}$ for $j=1,2, \cdots, k$ in the bounds in equation (19) in step 2 , we get exactly the bounds in equation (26) in step 3. Thus if we use FM elimination on this set of equations, from induction, we can get the bounds on

$$
d_{0}+\sum_{\mathcal{S}} d_{\mathcal{S}}
$$

as given in Theorem 3.1, where $\mathcal{S} \subset\{2,3, \ldots, k+1\}$, and bounds on $d_{j}$ for $j=1,2, \cdots, k$.

Compare the second group $((27))$ with the bounds in equation (19) in step 2, we can see if we change $\left(C_{0}-I_{j}\right)^{+}$in (19) to $\max \left\{I_{1}-I_{j+1}, C_{1}\right\}+\left(C_{0}-I_{1}\right)^{+}, d_{w_{j}}$ to $d_{w_{j+1}}$ for $j=1,2, \cdots, k$, and $d_{0}$ to $d_{0}+d_{1}$, we get exactly the bounds in equation (27) in step 3 . Thus if we use FM elimination on this set of equations, from induction we can get the bounds of

$$
d_{0}+d_{1}+\sum_{\mathcal{S}} d_{\mathcal{S}}
$$

as given in Theorem 3.1, where $\mathcal{S} \subset\{2,3, \ldots, k+1\}$.

Since these are all the bounds in Theorem 3.1, by induction, we have proven the result.

An alternative inner bound for the case when $\mathrm{SNR}_{i} \geq \mathrm{INR}_{i}$ is demonstrated in Section A.

\subsection{Proof of Theorem 3.2}

\subsubsection{The outer bound}

With $k=1$, the channel equations are given by

$$
\begin{aligned}
& Y_{0}=h_{00} X_{0}+Z_{0}, \\
& Y_{1}=h_{01} X_{0}+h_{11} X_{1}+Z_{1} .
\end{aligned}
$$

Under the condition that $\mathrm{SNR}_{0} \geq \mathbf{I N R _ { 1 }}$, we can use the argument presented in [3] and [5] to bound the capacity region of this network by the capacity region of a degraded broadcast channel with equations

$$
\begin{aligned}
& \tilde{Y}_{0}=\tilde{X}+Z_{0}, \\
& \widetilde{Y}_{1}=\widetilde{Y}_{0}+\widetilde{Z}_{1},
\end{aligned}
$$

where $\tilde{X}$ is subject to the power constraint

$$
\mathbb{E}\left[|\widetilde{X}|^{2}\right] \leq \mathrm{SNR}_{0}+\frac{\mathrm{SNR}_{1} \mathrm{SNR}_{0}}{\mathrm{INR}_{1}}
$$

and $\widetilde{Z}_{1} \sim \mathcal{C N}\left(0, \frac{\mathrm{SNR}_{0}}{\mathrm{INR}_{1}}-1\right)$ is independent of $Z_{0} \sim \mathcal{C N}(0,1)$. Hence, the outer bound on the original channel is given by the minimum of the point-to-point interference-free 
capacities of the links and the rates achievable in the associated broadcast channel, i.e., the union, over all $\alpha \in[0,1]$, of the regions

$$
\begin{aligned}
& R_{0}^{\text {outer }} \leq \log \left(1+\mathrm{SNR}_{0}\right) \\
& R_{0}^{\text {outer }} \leq \log \left(1+\alpha\left(\mathrm{SNR}_{0}+\frac{\mathrm{SNR}_{0} \mathrm{SNR}_{1}}{\mathrm{INR}_{1}}\right)\right) \\
& R_{1}^{\text {outer }} \leq \log \left(1+\mathrm{SNR}_{1}\right) \\
& R_{1}^{\text {outer }} \leq \log \left(1+\frac{(1-\alpha)\left(\mathrm{SNR}_{1}+\mathrm{INR}_{1}\right)}{1+\alpha\left(\mathrm{SNR}_{1}+\mathrm{INR}_{1}\right)}\right)
\end{aligned}
$$

For general $k>1$, we can augment the outer bound region above with the bounds $R_{i} \leq \log \left(1+\mathrm{SNR}_{i}\right)$, for $i=2,3, \ldots, k$ and call the resulting region by $\mathcal{O}_{1}$. This region is then certainly an outer bound to the capacity region of the network since all but the first interference link is set to zero. Since the capacity region of the network is contained in each of the regions $\mathcal{O}_{i}$, it is also contained in $\bigcap_{i=1}^{k} \mathcal{O}_{i}$.

\subsubsection{The inner bound}

Again, we start with the $k=1$ case. If the long-range user lowers its transmit SNR (by lowering its power) from SNR to $\gamma$ SNR, for some $\gamma \in[0,1]$, and the short-range receiver treats the interference as noise, the rate achieved by the two users is

$$
\begin{aligned}
& R_{0}^{\text {inner }} \leq \log \left(1+\gamma \mathrm{SNR}_{0}\right) \\
& R_{1}^{\text {inner }} \leq \log \left(1+\frac{\mathrm{SNR}_{1}}{1+\gamma \mathrm{INR}_{1}}\right)
\end{aligned}
$$

It is easy to check that the largest gap in $R_{1}$ between the outer and inner bounds, over all values of $R_{0}$, happens at the point where

$$
\alpha=\frac{\mathrm{INR}_{1}}{\left(1+\mathrm{SNR}_{1}\right)\left(\mathrm{SNR}_{1}+\mathrm{INR}_{1}\right)}
$$

in the outer bound and

$$
\gamma=\frac{1}{1+\mathrm{SNR}_{1}}
$$

in the inner bound. Furthermore, if $\mathrm{SNR}_{1} \geq \mathrm{INR}_{1}$, this gap is less than one bit at this point, therefore

$$
R_{1}^{\text {outer }}-R_{1}^{\text {inner }} \leq 1
$$

for all $R_{0} \in\left[0, \log \left(1+\mathrm{SNR}_{0}\right)\right]$ under this condition.

Now consider the case with $k>1$. To achieve any $R_{0}$, the long-range user lowers its power to $\gamma$ SNR for $\gamma \in[0,1]$. By the result shown above, if each of the short-range users treats the interference as noise, they are guaranteed to achieve a rate that is within one bit of the highest rate they can get in the absence of other short-range users. Since the presence of other short-range users cannot increase the capacity region, we conclude that each short-range user $i$ can achieve a rate within one bit of the boundary of the capacity region of the network in the $i$-th direction. Hence we have proved the theorem. 


\subsection{Proof of Theorem 3.3}

\subsubsection{The outer bound}

We first focus on the $k=1$ case, i.e., a network with a single short-range user. We can apply the outer bound on the capacity region of the network, found in Section 4.1.1 of [4]:

$$
\begin{aligned}
R_{0} & \leq\left(1+\mathrm{SNR}_{0}\right), \\
R_{1} & \leq\left(1+\mathrm{SNR}_{1}\right), \\
R_{0}+R_{1} & \leq \log \left(1+\mathrm{SNR}_{0}\right)+\log \left(1+\frac{\mathrm{SNR}_{1}}{1+\mathrm{INR}_{1}}\right) .
\end{aligned}
$$

for the regime in which $\mathrm{SNR}_{0} \geq I N R_{1}$.

The regime $\mathrm{SNR}_{0} \leq \mathrm{INR}_{1}$ corresponds to the "high-interference" regime and the exact capacity region is the classical result of [2]:

$$
\begin{aligned}
R_{0} & \leq\left(1+\mathrm{SNR}_{0}\right), \\
R_{1} & \leq\left(1+\mathrm{SNR}_{1}\right), \\
R_{0}+R_{1} & \leq \log \left(1+\mathrm{SNR}_{1}+\mathrm{INR}_{1}\right) .
\end{aligned}
$$

We use the high-SNR approximations to obtain the outer bound on the degree-of-freedom region of the $k=1$ network

$$
\begin{aligned}
d_{0} & \leq C_{0}, \\
d_{1} & \leq C_{1}, \\
d_{0}+d_{1} & \leq \max \left\{I_{1}, C_{1}\right\}+\left(C_{0}-I_{1}\right)^{+} .
\end{aligned}
$$

For the $k>1$ case, we first form $\mathcal{D}_{i}$ by removing all but the $i$-th interference link is contained in the following region

$$
\begin{aligned}
d_{0} & \leq C_{0}, \\
d_{j} & \leq C_{j}, \quad j=1,2, \ldots, k \\
d_{0}+d_{i} & \leq \max \left\{C_{0}, I_{i}\right\}+\left(C_{i}-I_{i}\right)^{+} .
\end{aligned}
$$

Specializing to the case when $\operatorname{SNR}_{i} \geq \operatorname{INR}_{i}\left(C_{i} \geq I_{i}\right)$ and $\mathrm{SNR}_{0} \geq \operatorname{INR}_{i}\left(C_{0} \geq I_{i}\right)$ for $i=1,2, \ldots, k$ and taking the intersection of the above regions over all $i=1,2, \ldots, k$, we obtain the outer bound which matches the statement of the theorem.

\subsubsection{The inner bound}

We assume that $I \mathrm{INR}_{1} \geq \mathrm{INR}_{2} \geq, \ldots$, INR $\mathrm{NR}_{k}$ (equivalently, $I_{1} \geq I_{2} \geq, \ldots, I_{k}$ ) without loss of generality, and we will refer to Fig. 3 since this is the shape of the degree-of-freedom region of each of the underlying $k=1$ networks of our problem. The approach taken is to maximize the linear functional

$$
d_{0}+\sum_{i=1}^{k} \mu_{i} d_{i}
$$




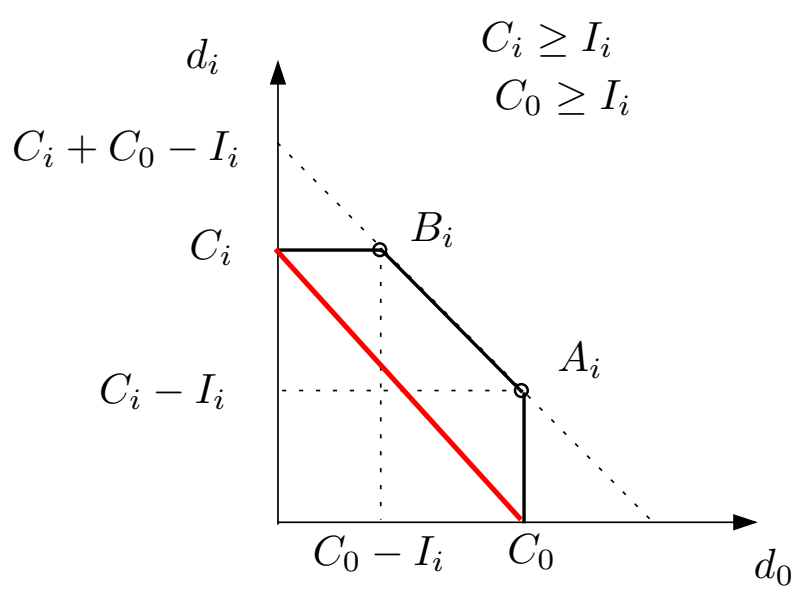

Figure 3: The shape of the degree-of-freedom region for the $k=1$ case, under weak interference. Orthogonalization is strictly suboptimal as shown by the straight line connecting $C_{i}$ and $C_{0}$.

over all achievable degree of freedom vectors $\left(d_{0}, d_{1}, \ldots, d_{k}\right)$.

Case 1: $\sum_{i=1}^{k} \mu_{i} \leq 1$. Define

$$
\epsilon_{i}:=\frac{\mu_{i}}{\sum_{j=1}^{k} \mu_{j}}
$$

for each $i=1,2, \ldots, k$. Then we can express the linear functional as

$$
d_{0}+\sum_{i=1}^{k} \mu_{i} d_{i}=\sum_{i=1}^{k}\left(\mu_{i} d_{i}+\epsilon_{i} d_{0}\right) .
$$

By the choice of $\epsilon_{i}$, each of the individual linear functionals $\left(\mu_{i} d_{i}+\epsilon_{i} d_{0}\right)$ is maximized at the corner point $A_{i}$ of Fig. 3 , where $d_{0}=C_{0}$ and $d_{i}=C_{i}-I_{i}$. These points are simultaneously achieved if each of the users $i=1,2, \ldots, k$ reduces its transmit power to SNR/INR $i$ so that the long-range user 0 can obtain its full degrees of freedom.

Case 2: $\sum_{i=1}^{k} \mu_{i}>1$.

$\mu_{1} \geq 1:$ Write

$$
d_{0}+\sum_{i=1}^{k} \mu_{i} d_{i}=\left(\mu_{1} d_{1}+d_{0}\right)+\sum_{i=1}^{k} \mu_{i} d_{i}
$$

and observe that the first linear functional $\left(\mu_{1} d_{1}+d_{0}\right)$ is maximized at corner point $B_{1}$ of Fig. 3, while each of the individual degrees of freedom is maximized at its point-to-point maximum degree of freedom $d_{i}=C_{i}$. The optimal scheme is for user 0 to treat all the interference as noise and all the short range users $i=1,2, \ldots, k$ to use full power. The dominant interference is due to user 1 which limits the degrees of freedom of user 0 to $d_{0}=C_{0}-I_{1}$. 
$\mu_{1}<1$ : This case is further broken into other cases:

$\mu_{2} \geq 1-\mu_{1}:$ Write

$$
\begin{aligned}
& d_{0}+\sum_{i=1}^{k} \mu_{i} d_{i}=\mu_{1}\left(d_{1}+d_{0}\right)+ \\
& \left(\mu_{2} d_{2}+\left(1-\mu_{1}\right) d_{0}\right)+\sum_{i=3}^{k} \mu_{i} d_{i}
\end{aligned}
$$

and observe that the second linear functional $\left(\mu_{2} d_{2}+\left(1-\mu_{1}\right) d_{0}\right)$ is maximized at the corner point $B_{2}$, where $d_{0}=C_{0}-I_{2}$ and $d_{2}=C_{2}$. This point is achieved by user 0 treating the interference of all other users as noise. User 1 can achieve the maximum sum of degrees of freedom $\left(d_{1}+d_{0}\right)$ at the point where $d_{0}=C_{0}-I_{2}$ and $d_{1}=C_{1}-\left(I_{1}-I_{2}\right)$ by lowering its power to $\operatorname{SNR}\left(\operatorname{INR}_{2} / \mathrm{INR}_{1}\right)$. Each of the users $i=3,4, \ldots, k$ achieves its individual point-to-point degree of freedom $d_{i}=C_{i}$.

$\mu_{2}<1-\mu_{1}$ : In this case, we have further cases

$\mu_{3} \geq 1-\left(\mu_{1}+\mu_{2}\right):$ Express the linear functional as

$$
\begin{aligned}
& d_{0}+\sum_{i=1}^{k} \mu_{i} d_{i}= \\
& \mu_{1}\left(d_{1}+d_{0}\right)+\mu_{2}\left(d_{2}+d_{0}\right)+ \\
& \left(\mu_{3} d_{3}+\left(1-\left(\mu_{1}+\mu_{2}\right) d_{0}\right)+\sum_{i=4}^{k} \mu_{i} d_{i} .\right.
\end{aligned}
$$

User 0 treats all interference as noise; users $i=3,4, \ldots, k$ achieve $d_{i}=C_{i}$; user 1 achieves $d_{1}=C_{1}-\left(I_{1}-I_{3}\right)$ by lowering its power to $\mathrm{SNR}\left(\mathrm{INR}_{3} / \mathrm{INR}_{1}\right)$; and user 2 achieves $d_{2}=C_{2}-\left(I_{2}-I_{3}\right)$ by lowering its power to $\mathrm{SNR}\left(\mathrm{INR}_{3} / \mathrm{INR}_{2}\right)$.

$\mu_{3}<1-\left(\mu_{1}+\mu_{2}\right)$ : Again, we have further branching into two cases: the case when $\mu_{4} \geq 1-\left(\mu_{1}+\mu_{2}+\mu_{3}\right)$ and the case when $\mu_{4}<$ $1-\left(\mu_{1}+\mu_{2}+\mu_{3}\right)$, which is then again split into two cases.

$\mu_{j} \geq 1-\left(\sum_{i=1}^{j-1} \mu_{i}\right):$ In general, at the $j$-th stage of the binary tree, for $j=2,3, \ldots, k$, we use the linear functional decomposition given by

$$
\begin{aligned}
& \sum_{i=1}^{j-1} \mu_{i}\left(d_{i}+d_{0}\right)+\left(\mu_{j} d_{j}+\left(1-\sum_{i=1}^{j-1} \mu_{i}\right) d_{0}\right) \\
& +\sum_{i=j+1}^{k} \mu_{i} d_{i} .
\end{aligned}
$$

The optimal scheme is for user 0 to treat all interference as noise; the users $i=j, j+1, \ldots, k$ to use full power to achieve maximum degrees 
of freedom $d_{i}=C_{i}$; and the users $i=1,2, \ldots, j-1$ to lower their power to $\operatorname{SNR}\left(\mathrm{INR}_{j} / \mathrm{INR}_{i}\right)$ to achieve $d_{i}=C_{i}-\left(I_{i}-I_{j}\right)$, respectively. The process continues in this fashion until the last case $\mu_{k} \geq 1-$ $\sum_{i=1}^{k-1} \mu_{i}$ is reached. At this point the bifurcation terminates because the condition is true by assumption. The proof is illustrated in Fig. 4.

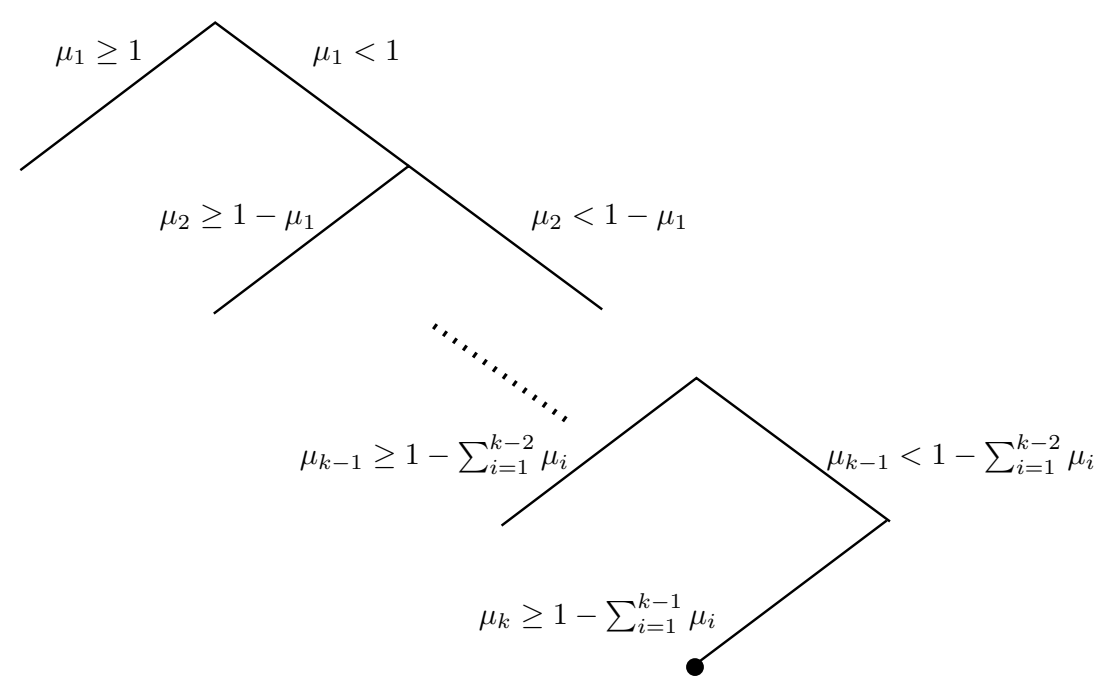

Figure 4: The case tree that illustrates the proof of the achievable scheme of Theorem 3.3.

\section{The "Strong-Interference" Regime}

If the condition $\mathrm{SNR}_{0} \leq \mathrm{INR}_{i}$ is met for all $i=1,2, \ldots, k$, in network of Fig. 2 (a), the network is in the so-called "strong-interference" regime and the capacity region can be exactly determined:

$$
\begin{aligned}
R_{0} & \leq\left(1+\mathrm{SNR}_{0}\right), \\
R_{i} & \leq\left(1+\mathrm{SNR}_{i}\right), \\
R_{0}+R_{i} & \leq\left(1+\mathrm{SNR}_{i}+\mathrm{INR}_{i}\right), \quad i=1,2, \ldots, k .
\end{aligned}
$$

This is a direct extension of the classical result in [2].

The optimal scheme is for the short-range transmitters to perform rate-splitting, adjusted to the rate $R_{0} \leq\left(1+\mathrm{SNR}_{0}\right)$ selected by the long-range transmitter. In this scheme, each short-range transmitter performs superposition coding and the short-range receiver successively decodes and cancels-off the high-powered, low-rate codeword of its transmitter, followed by the codeword of the long-range transmitter, and then followed by the low-power, high-rate codeword of its transmitter. In this way, each short-range user can operate on the boundary of the capacity region of the channel that it would experience in the absence of the other transmitters. 


\section{Acknowledgement}

We would like to thank Prof. David Tse for his very useful comments, especially those regarding Theorem 3.1.

\section{A An alternative achievable scheme for Theorem 3.1 when $\mathrm{SNR}_{i} \geq \mathrm{INR}_{i}$}

In this section we describe an alternative achievable scheme which achieves the degreeof-freedom region given in Theorem 3.1 under the assumption that $\mathrm{SNR}_{i} \geq \mathrm{INR}_{i}$. Our approach is to maximize the linear functional

$$
d_{0}+\sum_{i=1}^{k} \mu_{i} d_{i}
$$

over all achievable degree of freedom vectors $\left(d_{0}, d_{1}, \ldots, d_{k}\right) \in \mathcal{D}\left(\left\{\mathrm{SNR}_{i}\right\}_{i=0}^{k},\left\{\mathrm{INR}_{i}\right\}_{i=1}^{k}\right)$.

\section{A.1 The "weak interference" regime: $\mathrm{SNR}_{0} \geq \mathrm{INR}_{i}$}

Case 1: $\sum_{i=1}^{k} \mu_{i} \leq 1$. Define

$$
\epsilon_{i}:=\frac{\mu_{i}}{\sum_{j=1}^{k} \mu_{j}}
$$

for each $i=1,2, \ldots, k$. Then we can express the linear functional as

$$
d_{0}+\sum_{i=1}^{k} \mu_{i} d_{i}=\sum_{i=1}^{k}\left(\mu_{i} d_{i}+\epsilon_{i} d_{0}\right) .
$$

By the choice of $\epsilon_{i}$, each of the individual linear functionals $\left(\mu_{i} d_{i}+\epsilon_{i} d_{0}\right)$ is maximized at the corner point $A_{i}$ of Fig. 3, where $d_{0}=C_{0}$ and $d_{i}=C_{i}-I_{i}$. These points are simultaneously achieved if user 0 transmits at full power and each of the users $i=1,2, \ldots, k$ treats the interference from user 0 as noise.

Case 2: $\sum_{i=1}^{k} \mu_{i}>1$. Suppose that $\mathrm{INR}_{1} \geq \mathrm{INR}_{2} \geq \ldots \geq \mathrm{INR}_{k}$, which can be assumed without loss of generality.

$\mu_{1} \geq 1$ : Write

$$
d_{0}+\sum_{i=1}^{k} \mu_{i} d_{i}=\left(\mu_{1} d_{1}+d_{0}\right)+\sum_{i=1}^{k} \mu_{i} d_{i}
$$

and observe that the first linear functional $\left(\mu_{1} d_{1}+d_{0}\right)$ is maximized at corner point $B_{1}$ of Fig. 3, while each of the individual degrees of freedom is maximized at its point-to-point maximum degree of freedom $d_{i}=C_{i}$. The optimal scheme is for user 0 to reduce its power to $\mathrm{SNR} / \mathrm{INR}_{1}$ and each of the other users to treat the interference as noise. 
$\mu_{1}<1$ : This case is further broken into other cases:

$\mu_{2} \geq 1-\mu_{1}:$ Write

$$
d_{0}+\sum_{i=1}^{k} \mu_{i} d_{i}=\mu_{1}\left(d_{1}+d_{0}\right)+\left(\mu_{2} d_{2}+\left(1-\mu_{1}\right) d_{0}\right)+\sum_{i=3}^{k} \mu_{i} d_{i}
$$

and observe that the second linear functional $\left(\mu_{2} d_{2}+\left(1-\mu_{1}\right) d_{0}\right)$ is maximized at corner point $B_{2}$, where $d_{0}=C_{0}-I_{2}$ and $d_{2}=C_{2}$. If user 0 lowers its power to SNR/INR , then by treating interference as noise, the point $B_{2}$ is achieved for user 2 , User 1 can achieve the maximum total degrees of freedom $\left(d_{1}+d_{0}\right)$ at the point where $d_{0}=C_{0}-I_{2}$ and $d_{1}=C_{1}-\left(I_{1}-I_{2}\right)$ and each of the users $i=3,4, \ldots, k$ achieves its individual point-to-point degree of freedom $d_{i}=C_{i}$.

$\mu_{2}<1-\mu_{1}$ : In this case, we have further cases

$\mu_{3} \geq 1-\left(\mu_{1}+\mu_{2}\right)$ : Express the linear functional as

$$
\begin{aligned}
& d_{0}+\sum_{i=1}^{k} \mu_{i} d_{i}= \\
& \mu_{1}\left(d_{1}+d_{0}\right)+\mu_{2}\left(d_{2}+d_{0}\right)+\left(\mu_{3} d_{3}+\left(1-\left(\mu_{1}+\mu_{2}\right) d_{0}\right)+\sum_{i=4}^{k} \mu_{i} d_{i} .\right.
\end{aligned}
$$

User 0 reduces power to $\mathrm{SNR} / \mathrm{INR}_{3}$ and all the other users treat interference as noise to achieve $d_{i}=C_{i}$, for $i=3,4, \ldots, k, d_{1}=$ $C_{1}-\left(I_{1}-I_{3}\right)$ and $d_{2}=C_{2}-\left(I_{2}-I_{3}\right)$.

$\mu_{3}<1-\left(\mu_{1}+\mu_{2}\right)$ : Again, we have further branching into two cases: the case when $\mu_{4} \geq 1-\left(\mu_{1}+\mu_{2}+\mu_{3}\right)$ and the case when $\mu_{4}<$ $1-\left(\mu_{1}+\mu_{2}+\mu_{3}\right)$, which is then again split into two cases.

$\mu_{j} \geq 1-\left(\sum_{i=1}^{j-1} \mu_{i}\right):$ In general, at the $j$-th stage of the binary tree, for $j=2,3, \ldots, k$, we use the linear functional decomposition given by

$$
\sum_{i=1}^{j-1} \mu_{i}\left(d_{i}+d_{0}\right)+\left(\mu_{j} d_{j}+\left(1-\sum_{i=1}^{j-1} \mu_{i}\right) d_{0}\right)+\sum_{i=j+1}^{k} \mu_{i} d_{i}
$$

The optimal scheme is for user 0 to reduce its power to $\mathrm{SNR}_{\mathbf{I N R}}$ to achieve $d_{0}=C_{0}-I_{j}$. By treating interference as noise, the users $i=j, j+1, \ldots, k$ get degrees of freedom $d_{i}=C_{i}$ and the users $i=$ $1,2, \ldots, j-1$ achieve $d_{i}=C_{i}-\left(I_{i}-I_{j}\right)$.

The process continues in this fashion until the last case $\mu_{k} \geq 1-$ $\sum_{i=1}^{k-1} \mu_{i}$ is reached. At this point the bifurcation terminates because the condition is true by assumption. The proof is illustrated in Fig. 4. 


\section{A.2 The "strong interference" regime: $\mathrm{SNR}_{0} \leq \mathrm{INR}_{i}$}

Case 1: $\sum_{i=1}^{k} \mu_{i} \leq 1$. As in Case 1 of Section A.1, we can decompose the linear functional into the form given in (32). In this case, each of the linear functionals $\left(\mu_{i} d_{i}+\epsilon_{i} d_{0}\right)$ is maximized at corner point $A_{i}$, for $i=1,2 \ldots, k$. These points are achieved when user 0 transmits at full power to get a degree of freedom of $d_{0}=C_{0}$ and when each of the other users performs superposition coding (a.k.a. power splitting or rate splitting), to obtain $d_{i}=C_{i}-C_{0}$. The following are the details of this scheme:

1. User $i$, breaks its codeword into two parts and performs superposition coding so that the overall codeword is $X_{i}=X_{u, i}+X_{w, i}$, with individual powers $P_{u, i}=\left(\mathrm{SNR} \mathrm{INR}_{1}\right) /\left(\mathrm{SNR}_{0} \mathrm{SNR}_{i}\right)$ and $P_{w, i}=\mathrm{SNR}$.

2. The codeword $X_{w, i}$ is decoded first, subtracted off, then the interfering codeword of user $0, X_{0}$, is decoded and subtracted off, and finally the codeword $X_{u, i}$ is decoded.

Case 2: $\sum_{i=1}^{k} \mu_{i}>1$. As in Case 1 above, the linear functional can be decomposed into form (32). Each of the linear functionals $\left(\mu_{i} d_{i}+\epsilon_{i} d_{0}\right)$ is then maximized at the same corner point where $d_{0}=0$, i.e., the long range user is shut-off. Hence, all the short-range users can achieve their point-to-point maximum degrees of freedom $d_{i}=C_{i}, i=1,2, \ldots, k$.

\section{A.3 The heterogenous interference regime}

Suppose that

$$
\begin{aligned}
& \mathrm{SNR}_{0} \leq \mathrm{INR}_{i} \text { for } i=1,2, \ldots, j, \text { and } \\
& \mathrm{SNR}_{0} \geq \mathrm{INR}_{i} \text { for } i=j+1, j+2, \ldots, k,
\end{aligned}
$$

and that $I_{N R} R_{j+1} \geq I N R_{j+2} \geq \ldots \geq I N R_{k}$, both of which can be assumed without loss of generality. Then, we have the familiar cases:

Case 1: $\sum_{i=1}^{k} \mu_{i} \leq 1$. This case is similar to Case 1 in Section A.2: user 0 uses full power to achieve $d_{0}=C_{0}$; users $i=1,2, \ldots, j$ perform superposition coding (power splitting or rate splitting) to achieve $d_{i}=C_{i}-C_{0}$; and users $i=j+1, j+2, \ldots, k$ simply treat interference as noise to achieve $d_{i}=C_{i}-I_{i}$.

Case 2: $\sum_{i=1}^{k} \mu_{i}>1$. In this situation we have two subcases:

Subcase 1: There exists at least one $i \in\{1,2, \ldots, j\}$ such that $\mu_{i} \geq 1$. Choose one such index and call it $i_{*}$. Then we can express the linear functional as

$$
\left(d_{i_{*}}+d_{0}\right)+\left(\mu_{i_{*}}-1\right) d_{i_{*}}+\sum_{i \neq i_{*}} \mu_{i} d_{i} .
$$

Each of the terms in the above linear functional is maximized at the point where $d_{0}=0$, i.e., the long range user is shut off, and each of the users $i=1,2, \ldots, k$ achieves its point-to-point degree of freedom $d_{i}=C_{i}$. 
Subcase 2: $\mu_{i}<1$ for all $i \in\{1,2, \ldots, j\}$. In this case we further have two subcases:

$\mu_{j+1} \geq 1-\sum_{i=1}^{j} \mu_{i}:$ We can decompose the linear functional as

$$
\sum_{i=1}^{j} \mu_{i}\left(d_{i}+d_{0}\right)+\left(\mu_{j+1} d_{j+1}+\left(1-\sum_{i=1}^{j} \mu_{i}\right) d_{0}\right)+\sum_{i=j+2}^{k} \mu_{i} d_{i} .
$$

If the long-range user lowers its power to $\mathrm{SNR} / \mathrm{SNR}_{j+1}$ (thus achieving a degree of freedom of $\left.d_{0}=C_{0}-I_{j+1}\right)$, user $j+1$ can treat interference as noise to achieve the degree of freedom $d_{j+1}=C_{j+1}$ at corner point $B_{j+1}$. With such a choice of $d_{0}$, the linear functionals $\left(d_{i}+d_{0}\right)$ for $i=1,2, \ldots, j$ are maximized at the point where users $1,2, \ldots, j$ achieve degrees of freedom $d_{i}=C_{i}-\left(C_{0}-I_{j+1}\right)$. These degrees of freedom are achieved if the users $i=1,2, \ldots, j$ perform superposition coding as described in Case 1 of Section A.2. Finally, the users $j+2, j+3, \ldots, k$ achieve their pointto-point maximum degrees of freedom $d_{i}=C_{i}$ by treating interference as noise.

$\mu_{j+1}<1-\sum_{i=1}^{j} \mu_{i}$ : In this case, we have further cases:

$\mu_{j+2} \geq 1-\sum_{i=1}^{j+1} \mu_{i}$ : Write the linear functional as

$$
\sum_{i=1}^{j+1} \mu_{i}\left(d_{i}+d_{0}\right)+\left(\mu_{j+2} d_{j+2}+\left(1-\sum_{i=1}^{j+1} \mu_{i}\right) d_{0}\right)+\sum_{i=j+3}^{k} \mu_{i} d_{i}
$$

and repeat the analysis that was used in the case when $\mu_{j+1}>1-$ $\sum_{i=1}^{j} \mu_{i}$. This time, $d_{j+2}=C_{j+2}$ at the corner point $B_{j+2}, d_{i}=C_{i}$ for $i=j+3, j+4, \ldots, k$, both being achieved by treating interference as noise. Similarly, $d_{i}=C_{i}-\left(C_{0}-I_{j+1}\right)$ is achieved by superposition coding for $i=1,2, \ldots, j$ and $d_{j+1}=C_{j+1}-\left(I_{j+1}-I_{j+2}\right)$ which is achieved by treating interference as noise.

$\mu_{j+2}<1-\sum_{i=1}^{j+1} \mu_{i}$ : In this case we again have two subcases depending on whether $\mu_{j+3}$ is larger or smaller than $1-\sum_{i=1}^{j+2} \mu_{i}$ : If it is larger, we are done, but if it is smaller, then we again bifurcate into two more cases. The process continues in this fashion until the last case $\mu_{k} \geq 1-\sum_{i=1}^{k-1} \mu_{i}$ is reached. At this point the bifurcation terminates because the condition is true by assumption (see Fig. 4).

\section{References}

[1] A.B. Carleial, "Interference channels," IEEE Transactions on Information Theory, vol. 24, pp. 60-70, January 1978.

[2] H. Sato, "The capacity of the Gaussian interference channel under strong interference," IEEE Transactions on Information Theory, vol. 27, pp. 786-788, Nov. 1981. 
[3] H.M. Costa, "On the Gaussian interference channel," IEEE Transactions on Information Theory, vol. 31, pp. 607-615, Sept. 1985.

[4] D. Tse, R. Etkin, and H. Wang, "Gaussian interference channel capacity to within one bit," submitted to IEEE Transactions on Information Theory, February 2007, http://arxiv.org/abs/cs/0702045.

[5] H. Sato, "On degraded Gaussian two-user channels" (corresp.), IEEE Transactions on Information Theory, vol. 24, pp. 638-640, September 1978.

[6] T.S. Han and K. Kobayashi, "A new achievable rate region for the interference channel," IEEE Transactions on Information Theory, vol. 27, pp. 49-60, January 1981. 